\title{
Elastic-Microplastic Transitions in Rocks during Low-Intensity Wave Propagation
}

\author{
By Eduard Mashinskii*
}

The field and laboratory acoustic experiments have been conducted for the study of nonstandard (microplastic) inelasticity in loam and siltstone. New data was obtained thanks to seismic/acoustic recording in the form of "the stress - time" with the microsecond resolution. Unusual inelastic manifestations represented in the form of stress plateau, the stress drop and the steepness decrease on wave front complicate wave form. The short-term discrepancy of the upward and downward stress course in some time interval is characterized as micro-hysteresis effect. The microplastic process influences the wave propagation, transforms its form and dynamic parameters. The observed effects depend on the magnitude of the applied dynamic stress. The discrete inelasticity plays an important part not only in wave dynamics but can exerts influence on global deformation processes in the Earth.

Keywords: Amplitude dependence of wave velocity and attenuation, Deformation and time delay, Inelastic seismic parameters, Inelasticity, Relaxation spectra of wave attenuation, Rock's microplasticity.

\section{Introduction}

An important task in geodynamics, seismology and seismic prospecting is the study of deformation mechanisms in rocks. Deformation processes in rocks occur under the action of natural and man-caused sources, and cover a wide deformation range $\left(10^{-12}--10^{-2}\right)$ and a still greater range of strain rate. The study of the dynamic deformation processes enables to obtain useful information about a geological medium that is required for a solution of many problems.

One of important tasks in seismology and seismic prospecting is the determination of a wave attenuation physical mechanism taking into account a new knowledge about the discrete (intermittent) inelasticity of rocks that have been recently obtained during of the nonstandard experiments (Mashinskii, $2012 ; 2014 ; 2016)$. At present time in the theoretical researchs, the intermittent deformation process in rocks under the action of low-intensity stress is disregarded, and the mechanical model for rock is not has a discrete deformation element. Known viscoelastic model (standard linear solid) is insufficient for explaining of some effects, for example like the amplitude dependence of wave velocity and attenuation detected earlier in the seismic and acoustic frequency range (Mashinskii et al., 1999; Mashinskii, 2004; 2007a,b; 2008; Zaitsev et al., 1999). It is expected that amplitude-dependent effects can be a perspective for problem solving in applied seismology and in other science area. The in itself

${ }^{*}$ Researcher, Trofimuk Institute of Petroleum Geology and Geophysics, Siberian Branch of the RAS, Russia. 
presence of the amplitude dependence of the seismic and acoustic parameters implies a possible existence of some additional attenuation mechanism besides already known mechanisms. The latest researches in this area have shown that there really are the explicit data that can be connected with unusual inelasticity type.

The first data in geophysics about unusual inelasticity type in the form of microplasticity have been obtained in quasi-static experiments on the samples of various natural materials (sandstone, siltstone, mudstone, quartz and others) (Mashinsky, 1994; Mashinskii, 2005b). A new type of rock inelasticity was termed by 'microplasticity' by analogy with solid-state physics in which microplasticity is well-known fact for metals, alloys and others materials but not for rocks. In contrast to macro-plasticity, microplasticity presents "plasticity insets" within an elastic region where stresses and strains are very small $\left(\sim 10^{-8}-10^{-6}\right)$. Microplasticity can be regarded as the plasticity in a microscopic scale (and even in a nanoscale) at localized regions, for example on grain boundaries. Substantively, microplasticity interrupts at short time an elastic process during loading or unloading. Here, the stress-strain ratio $\sigma(\varepsilon)$ depends on the joint contribution of the elastic and microplastic components in the total deformation. Microplasticity process on very small strains occurs owing to the stress concentration in the local areas of material. Thus the microplastic insets during stress accompany the elastic deformation course. The main mechanism of microplasticity in solids is associated with the motion of dislocations that causes intermittent micro/nano-plastic flow and residual deformation. As for rocks containing minerals with a high content of mobile dislocations, the mechanism of microplasticity may be the same as in other solids. Since the structure of rocks is much more complicated than in metals and alloys, several mechanisms of microplasticity are possible.

Dynamic processes study in rocks, in view of microplasticity existence, can give a chance obtain more valid data about a microstructure of rocks. Microplasticity presence reasonably explains contradictions between diverse data about amplitude dependence of seismic parameters. For example, generally accepted data show a decrease of wave velocity and the attenuation increase with increasing strain amplitude (Mavko, 1979; Winkler et al., 1979; Tutuncu et al., 1994; Ostrovsky and Johnston, 2001). However, some data show on the contrary an unusual increase of wave velocity and the decrease in attenuation with increasing strain amplitude (Johnston and Toksoz, 1980; Mashinskii et al., 1999; Mashinskii, 2004; Mashinskii, 2007a; Zaitsev et al., 1999). There are also indirect evidences of the unusual behavior of the elastic modulus in the dependence on the applied stress value (Mashinsky, 1994; Mashinskii, 2005b). It is shown that thanks to microplasticity, modulus can both decrease and increase in compliance with the curvature in the stress-strain ratio $\sigma(\varepsilon)$. This becomes possible because microplasticity contribution can both increase and decrease with increasing stress. There are theoretical works that also confirm the connection of curvature in the stress-strain ratio $\sigma(\varepsilon)$ with elastic modulus behavior (McCall and Guyer, 1994; Guyer et al., 1995; Guyer and Johnson, 1999). 
Unusual behavior of wave velocity in dependence on the strain amplitude value has been detected in dolomites in a laboratory experiment (Mashinskii and D'yakov, 1999; Mashinskii, 2004). Wave velocity with increasing the strain amplitude increases instead of usual it decrease that was established earlier. This result was also confirmed by observations in situ (Mashinskii et al., 1999; Zaitsev et al., 1999; Mashinskii, 2007b). A significant wave attenuation decrease due to an amplitude increase in the $\mu$-strain range was detected in sandstone, smoky quartz under confining pressure of $20 \mathrm{MPa}$ (Mashinskii, 2005a). The experiments conducted on the dry and saturated sandstone showed that attenuation in comparison with velocity is more sensitive to the amplitude variations (Mashinskii 2006, 2007a). These facts confirm the possibility of participation of microplasticity in the wave propagation process. The knowledge of dynamic microplasticity in geological material is limited, and until now not studied profoundly. In the present time, this study is going on in the experimental and the theoretical plan (Xu et al., 1998; Braccini et al., 2000; Mashinskii, 2008; 2010; Yarushina and Podladchikov, 2008; 2010; Derlet and Maaf, 2013).

The study of dynamic microplasticity in rocks in contrast to the study of static microplasticity (Mashinsky, 1994; Mashinskii, 2005b) has been conducted relatively recently. The first estimate of the microplasticity manifestations on seismic records was made by means of borehole-to-borehole survey (Mashinskii, 2010). During pulse propagation with different amplitudes, a shift of the arrival time and the pulse front protraction has been detected. A detailed description of a transformation mechanism of the pulse shape that takes place owing to microplasticity process in loam was made in the works (Mashinskii, 2012; 2014). There is the difference in microplasticity manifestation on seismic records received by using of seismic sources of positive and negative polarity. In these articles, large attention is also given to the seismic arrival. Microplasticity manifestations caused by acoustical wave of the $\mathrm{kHz}$ frequency range are detected in the sample of artificial sandstone in the laboratory experiment (Mashinskii, 2016).

This paper presents the new data obtained for loams and the results of laboratory testing of the siltstone sample in the acoustic frequency range. The experiment purpose is to examine the microplasticity effect in the siltstone sample and the comparison of these results with available data.

\section{Experimental Data}

\section{Method and Equipment}

In this paper, the results of two alternative experiments (field and laboratory) are presented, which show the manifestations of dynamic microplasticity for two types of rocks and under substantially different observation conditions. In both cases, the techniques and the range of relative deformations are the same. Despite these differences, here it is important for us to compare only the qualitative characteristics of microplasticity manifestations obtained in different 
frequency ranges. Our measurements are relative, since the evaluation of the microplasticity peculiarity is carried out by comparing of the pulse records with different amplitudes among themselves.

The field experiments were conducted in the proving ground not far from Novosibirsk. The upper part of the geological section is comparatively homogeneous and is composed of loams several tens of meters in thickness. Such medium is typical at shallow depth, i.e. it is usual low-velocity zone in seismic exploration. Loams are partially water-saturated up to $8.5 \mathrm{~m}$ with compressional wave velocity $\mathrm{Vp}=240-300 \mathrm{~m} / \mathrm{s}$. Below the depth of $\sim 9 \mathrm{~m}$ the wave travels in completely water-saturated loams with $\mathrm{Vp}=1500 \mathrm{~m} / \mathrm{s}$.

The measuring instruments were mounted in boreholes with diameter 110 $\mathrm{mm}$ and a depth of $12 \mathrm{~m}$, a distance between boreholes is $7 \mathrm{~m}$. A pulse source was located in one of the boreholes, and the receiver was placed in the other borehole. Seismic measurements were performed when the source and the receiver were at the same depth: $10 \mathrm{~m}-10 \mathrm{~m}$ (the completely saturated loams). The seismic source consists of a set of piezoelectric disks installed in a cylinder in the borehole. This is a cylinder in a borehole. The signal goes through a liquid spacer and hermetic elastic jacket contacting the borehole wall. The predominant pulse frequency recorded by receiver in the $10 \mathrm{~m}$ depth is about $450 \mathrm{~Hz}$.

The pressure receiver has a sensor of the piezoelectric type and, therefore, records compression waves. The receiver is connected with the borehole wall by an elastic spacer with a liquid. It ensures good contact of the seismic source and the receiver in the loam. The record is the stress as a function of the time, $\sigma(t)$. The signals were recorded using a time quantization step $\mathrm{t}_{\text {quan }}=8 \mu \mathrm{s}$. An amplitude quantization step $\left(\mathrm{A}_{\text {quan }}\right)$ in $\mathrm{ADC}$ equals $78 \mu \mathrm{V}$. The amplitude magnitude in volts was transformed to a magnitude of stress (Pascal) using the piezoelectric conversion ratio $\mathrm{K}_{\text {trans }}=100 \mu \mathrm{V} / \mathrm{Pa}$. There is a preamplifier with the amplification coefficient $\mathrm{K}=100$. The signals were recorded in the digital form of high-resolution during time (Bordo-B-421 system), and were processed on a computer.

The seismic and acoustic signals measurement procedure for both field and laboratory studies is the same. In our study, the magnitude of the wave amplitude is not constant. We record seismic and acoustic signals at several amplitude values. At a constant source-receiver location, the value of the amplitude in the source increases discretely to a certain maximum, and then decreases in the same order to the initial amplitude value. In accordance with this course, we register a passing wave. In the field experiment, the discrete change in amplitude value was fulfilled in a closed cycle, from the minimum amplitude value to the maximum and back to minimum, where: $A_{\min }=A_{1} \rightarrow A_{2} \rightarrow A_{3} \rightarrow$ $\mathrm{A}_{4}=\mathrm{A}_{\max } \rightarrow \mathrm{A}_{3} \rightarrow \mathrm{A}_{2} \rightarrow \mathrm{A}_{1}$. Thus, we have 7 records, of which 4 records were received during the increase in amplitude (so-called upward records) and 3 records (so-called downward records) were received while the amplitude was decreasing. Upward and downward amplitude values are equal: $\mathrm{A}_{1 \text {-up }}=\mathrm{A}_{1 \text {-down }}$, $\mathrm{A}_{2 \text {-up }}=\mathrm{A}_{2 \text {-down }}, \mathrm{A}_{3 \text {-up }}=\mathrm{A}_{3 \text {-down }}$. The relative amplitude increase is $\mathrm{A}_{2} / \mathrm{A}_{1}=1.4$, $\mathrm{A}_{3} / \mathrm{A}_{1}=2.1, \mathrm{~A}_{4} / \mathrm{A}_{1}=2.7$. Since recordings with the up and down amplitudes 
carry out in the quite equal conditions, it allows to obtain useful information, for example, about their repeatability or vice versa the discrepancy. In principle, a record with the down amplitude duplicates the upward amplitude record and let to compare measurements obtained with the same amplitude value.

Maximum stress and strain in a seismic pulse during its propagation were estimated approximately. As we deal with the pulse propagation, the strain amplitude is estimated relatively of the wave length, $\lambda$. The displacement amplitude $u$ is given by $\langle\ddot{u}\rangle_{t} / \omega^{2}$. Then $\varepsilon_{\max }=2 u / \lambda$. The maximum strain magnitude can be also estimated as (Adushkin et al., 1999; Mashinskii, 2005a): $\varepsilon_{\max }=v / V=2 \pi u / \lambda$ where $v$ is a particle velocity, $V$ is a wave velocity. The displacement is estimated using the transmission coefficient of piezoelectric transducer at the source voltage. At the distance of 7 meters, for the most part the stress is in the limits from units and tens of Pascal to the first hundreds Pascal, the strain is in the range of about $10^{-8}-10^{-6}$. Thus, small stresses and strains take place in the considerable part of the way during wave propagation between boreholes.

The laboratory experiment was carried out using the reflection method. The confining pressure on the sample was $20 \mathrm{MPa}$. The sample was a cylinder with a height of $2 \mathrm{~cm}$ and a diameter of $4 \mathrm{~cm}$. The rock is a consolidated siltstone taken from a depth of $2560 \mathrm{~m}$. The density of the rock is $2.3 \mathrm{~g} \mathrm{~cm}^{-3}$ with an average porosity of $15 \%$. The experiments were conducted at a dryroom temperature (about $20^{\circ} \mathrm{C}$ ). The change in amplitude value was fulfilled also in a closed cycle, from the minimum to the maximum and back. Excitation and reception of ultrasonic pulses ( $\mathrm{f} \sim 1.0 \mathrm{MHz}$ ) are carried out by means of the piezoelectric elements that are rigidly fixed on the acoustic-delay layer. A time quantization step in the analog-digital converter is $t_{\text {quan }}=32.5 \mathrm{~ns}$. An amplitude quantization step $\left(\mathrm{A}_{\text {quan }}\right)$ in $\mathrm{ADC}$ equals $78 \mu \mathrm{V}$. We record stress as a function of time, Digital signals were recorded with accumulation (no more than 32), which raises the data adequacy. In the laboratory experiment, the discrete change in amplitude value was fulfilled also in a closed cycle, from the minimum amplitude value to the maximum and back to minimum: $\mathrm{A}_{\min }=\mathrm{A}_{1}$ $\rightarrow \mathrm{A}_{2} \rightarrow \mathrm{A}_{3} \rightarrow \mathrm{A}_{4} \rightarrow \mathrm{A}_{5} \rightarrow \mathrm{A}_{6}=\mathrm{A}_{\max } \rightarrow \mathrm{A}_{5} \rightarrow \mathrm{A}_{4} \rightarrow \mathrm{A}_{3} \rightarrow \mathrm{A}_{2} \rightarrow \mathrm{A}_{1}=\mathrm{A}_{\mathrm{min}}$ Here, we have 11 records, of which 6 records were received during the increase in amplitude and 5 records were received while the amplitude was decreasing. Upward and downward amplitude values are equal: $A_{1 \text {-up }}=A_{1 \text {-down }}, A_{2 \text {-up }}=A_{2 \text { - }}$ down $, A_{3 \text {-up }}=A_{3 \text {-down }}, A_{4 \text {-up }}=A_{4 \text {-down }}, A_{5 \text {-up }}=A_{5 \text {-down }}$. The amplitude increases as $\mathrm{A}_{2} / \mathrm{A}_{1}=2, \mathrm{~A}_{3} / \mathrm{A}_{1}=4, \mathrm{~A}_{4} / \mathrm{A}_{1}=6, \mathrm{~A}_{5} / \mathrm{A}_{1}=8, \mathrm{~A}_{6} / \mathrm{A}_{1}=10$. The strain amplitude is in the range about $10^{-8}-10^{-6}$.

Previous data about seismic microplasticity manifestation (SMM) were received both using excitation source with initial deformation of one type (compression or tension) and with sources of two types (compressive and tensile) (Mashinskii, 2010; 2012; 2014). In this article we present the new results of the field and laboratory study of microplastic effects that are obtained for natural materials. 


\section{Results}

In order to obtain the new information about dynamic deformation in geological medium, registration of the seismic and acoustical waves was conducted in the digital form using a micro/nanoscale recording and corresponding visual representation. Signal recording in such representation is required for analysis of microplasticity effect.

It is found that seismic (acoustical) pulse in separate sites is complicated by the distinctive amplitude changes. These complications are presented in the ladder form with the horizontal plateaus and the stress drop. Such insets presumedly connected with microplasticity interrupt the 'normal' stress course, and cause delay of elastic deformation.

\section{Field Experiment}

Seismic pulse recorded in loams in borehole at the depth $10 \mathrm{~m}$ is shown in Figure 1. This recording is obtained on $A_{1}$ and $A_{2}$ (upward and downward) amplitude values. Initial part of negative wave phase in the expanded scale is presented in inset. Microplasticity manifestation originality can be seen in Figure 2 in which are shown in detail the wave front fragments for amplitudes $\mathrm{A}_{1}$ and $\mathrm{A}_{2}$.

Figure 1. Seismic Pulse Recorded in Loams on Two Amplitude Values. Pulse Fragment in the Expanded Scale is shown in Inset

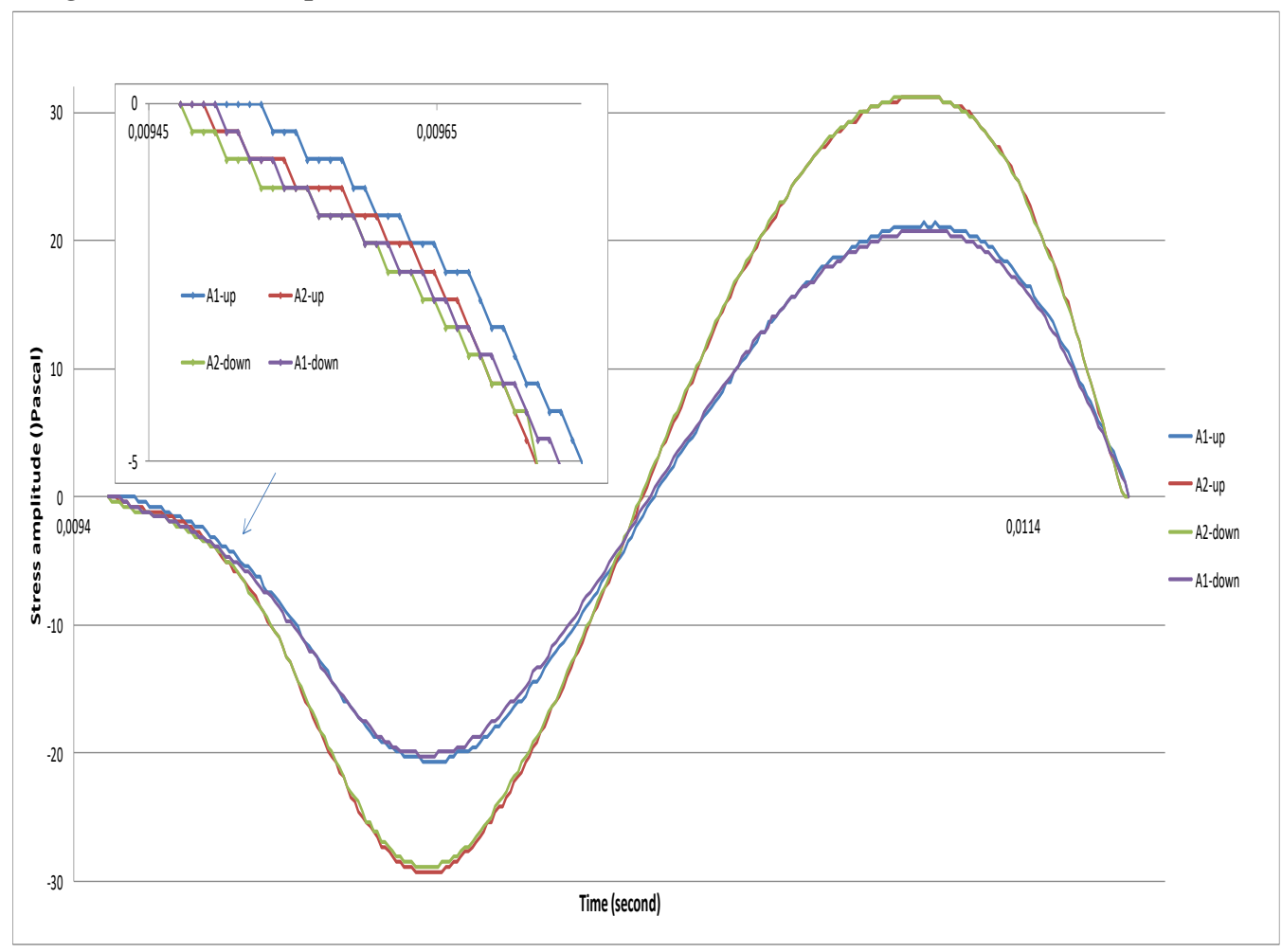


Figure 2. Wave Front Fragments with Amplitudes A1 and A2 in Loams

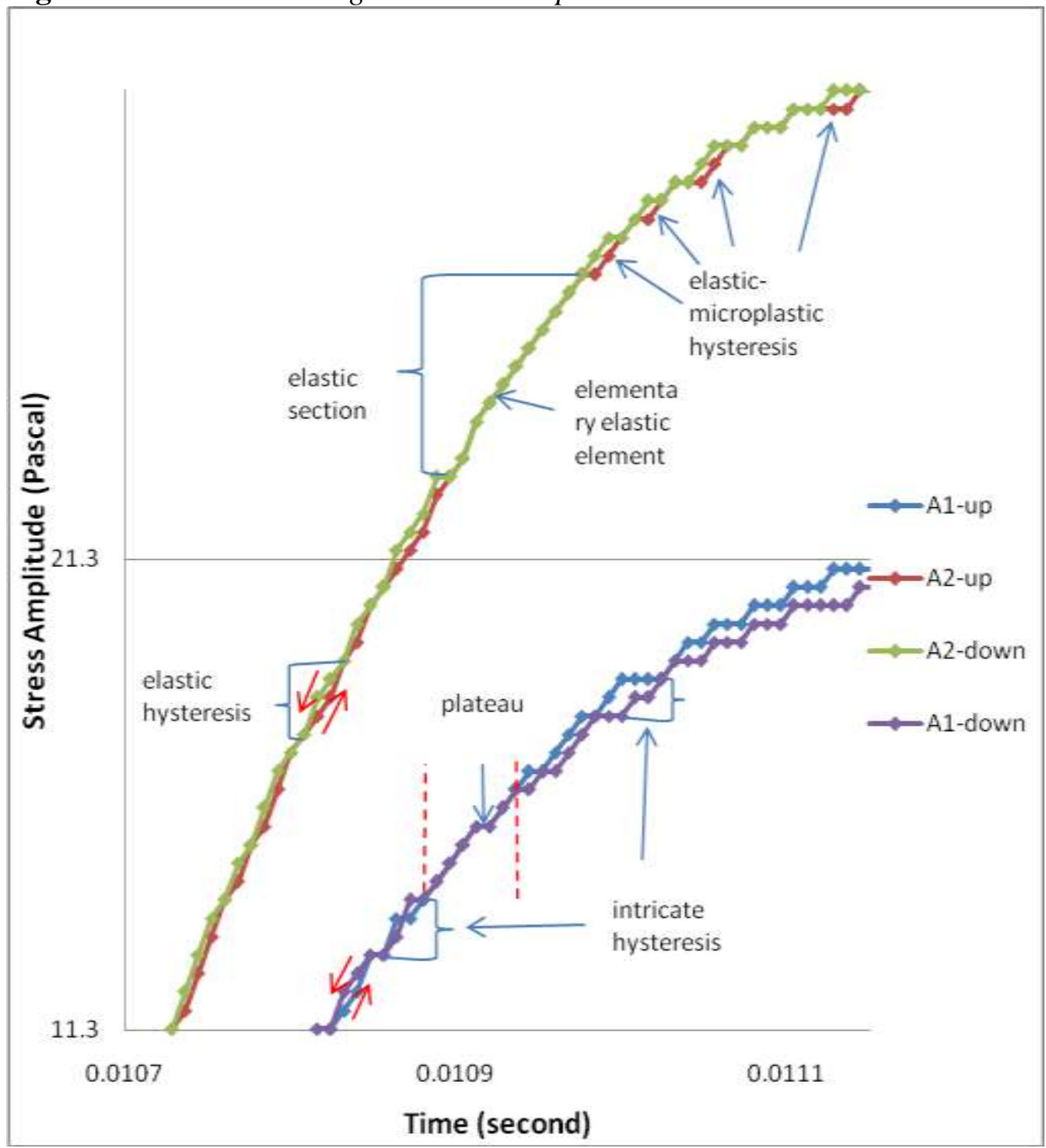

On wave front, there are the stress plateaus and other complications that are conceivably caused by microplastic inelasticity. The stress behavior during time depends on a joint action of the elastic and micro-plastic process. Comparison of the upward and downward trace shows the good repeatability of nonstandard manifestations. On wave front, there are areas in which takes place only the elastic deformation. Here, the $\sigma(t)$ curve presents the normal monotone increase in the stress amplitude (without complications), for example it can be seen in "elastic section" for amplitude A2. In the general case, plateaus of different duration can be situated both inside elastic section and be between elastic sections.

Complete identity of the upward and downward traces recorded on the equal amplitude value takes place not always. Besides, the complete coincidence of nonstandard manifestations on these traces occurs only in some time intervals. For example in Figure 2, it is a lengthy elastic section on the A2 trace and a lengthy elastic-microplastic section on the A1 trace marked by red dotted lines. 
There are also short time intervals of the elastic-microplastic behavior on the A1 and A2 traces with complete coincidence of two traces. However, the incomplete coincidence of two traces in the other time intervals does not mean the identity loss. In that case, the identity of two traces occurs on other level with original manifestations.

With stress increase during the time, we see the stress course divergence between the first and repeated traces, further these courses meet in the common point. This effect can be characterized as the micro-hysteresis on separate part of the $\sigma(t)$ curve. In Figure 2, we observe the hysteresis loops that arise repeatedly on wave front. When the hysteresis loop consists only of the elastic elements, it can be characterized as the elastic (viscous-elastic) hysteresis. If the hysteresis loop contains together the elastic and discrete-inelastic elements (plateaus), it is the elastic-microplastic hysteresis. As a rule, the hysteresis loop presents a geometrical figure in which takes place the qualitative and numerical equality of elements appurtenant to the elastic and discrete-inelastic process. In case of the elastic hysteresis, there is equality of the elementary elements with equal slope for the upward and downward branch of the hysteresis loop. For example, the elastic loop (marked by red arrows in Figure 2) with the duration of $3 \times \mathrm{t}_{\text {quan }}$ contains two elements with smaller steepness plus one element with bigger steepness. These upward-downward branches of the hysteresis loop looks together as rhomb in which parallel sides have the equal slope (microfront steepness in the $3 \times \mathrm{t}_{\text {quan }}$ time interval). The same situation takes place for the elastic-plastic hysteresis loop. Here, the quantity of the elastic and correspondingly discrete- inelastic elements in the upward loop branch equals the quantity of the same elements in its downward branch. It is observed not only for simple loops but also for the intricate hysteresis loops that perform the complicated trajectories (it is shown in Figure 2).

The pulse maximum of the same wave with amplitudes A1 and A2 in the expanded scale is presented for consideration in Figure 3. Here we also see the ladder-like stress behavior during time and numerous plateaus located close to the amplitude maximum. At the same time near of the maximum in pulse, the plateau duration increase is observed. 
Figure 3. Pulse Maximum on Amplitudes A1 and A2 in Loams

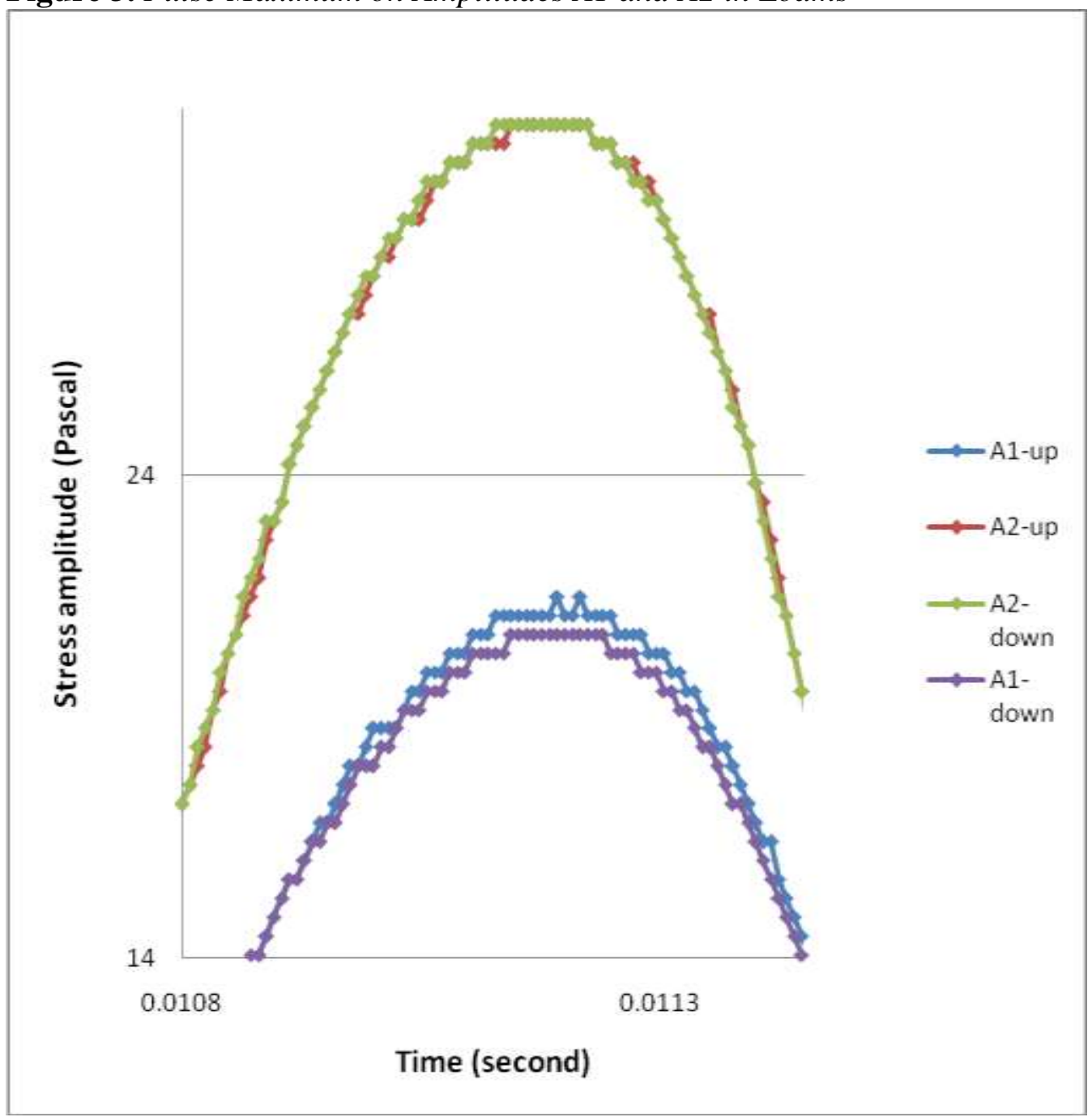

\section{Laboratory Experiment}

Acoustical pulse recorded in siltstone on six upward and downward amplitude values is presented in the analog form in Figure 4. The attentive examination of an analog signal reveals that a pulse form distortion is caused by numerous complications of an unknown nature. These complications in the form of highfrequency oscillations can be seen in the inset. Large-scale survey of pulse fragment presented in the digital representation in Figure 5 enables to understand the authentic nature of these complications.

Wave front on all amplitude values is complicated by the short-term disturbances in the form of the stress plateau and stress drop. As an example, some of complications in the form the stress drops are marked by the red dotted line. There are also separate manifestations in the form of the short-term decrease of the wave front steepness that occurs during one or more $t_{\text {quan }}$.

The so-called interchangeability effect of discrete-inelastic manifestations was detected thanks to the first and repeated (upward-downward) recording 
made for all amplitude values of propagating wave. Interchangeability implies the substitution possibility of the stress plateau in the one case on the stress drop, and in the other case vice versa the stress drop on the stress plateau in the same time interval. In our case, the interchangeability can be seen by means of the comparison of the upward and downward records obtained for the different amplitude values. Some examples are marked by two dotted lines in Figure 5. Such nonstandard manifestations are presumably related to the microplastic inelasticity (one of form it manifestation). This process along with the elastic process takes part in forming the dynamic characteristics of seismic and acoustical waves in rocks. In particular, such intermittent inelastic process distorts the wave form that holds information about geological medium.

Figure 4. Acoustical Pulse Recorded in Siltstone on Six Upward and Downward Amplitude Values

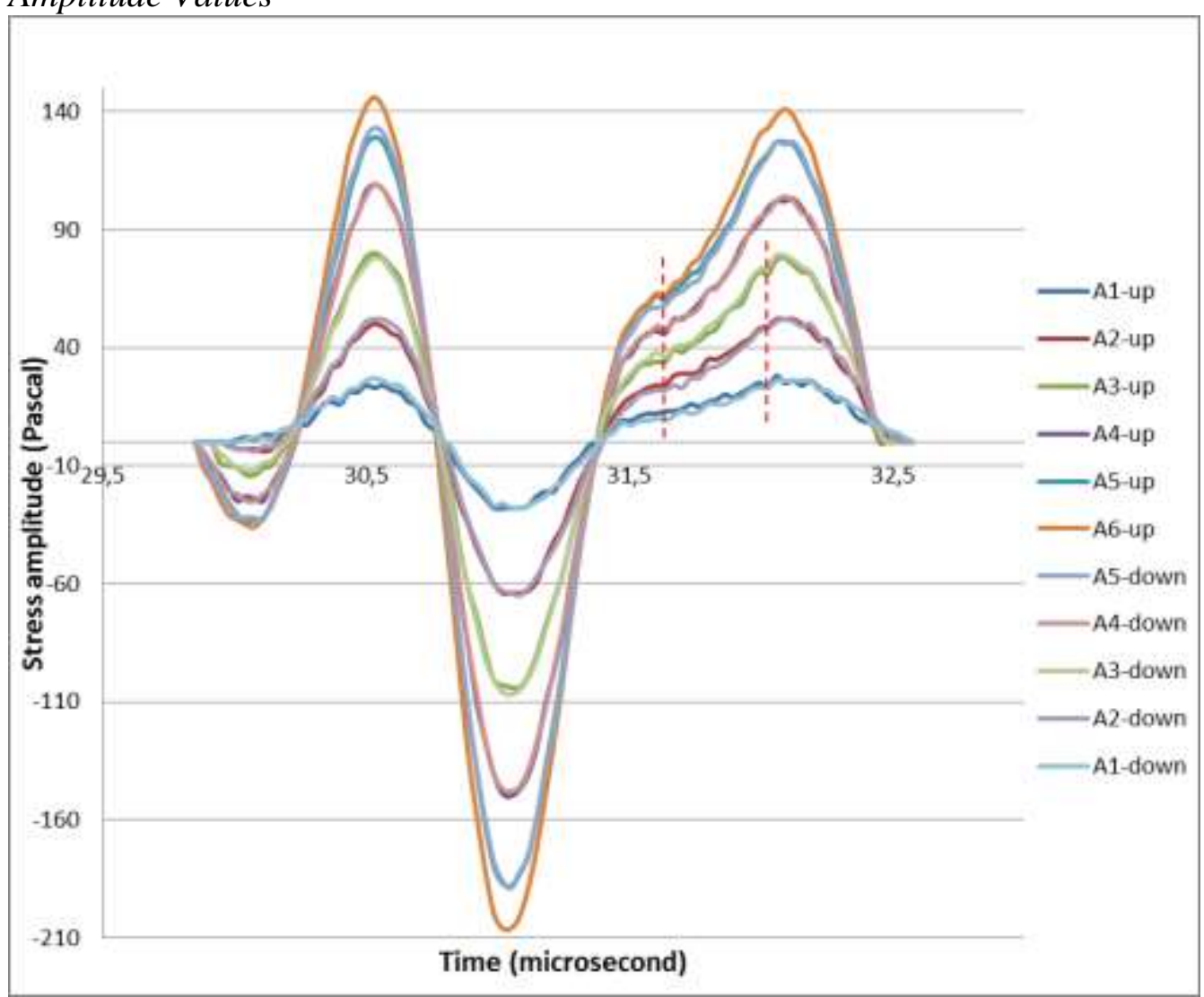


Figure 5. Pulse Fragment from Figure 4 in the Expanded Scale

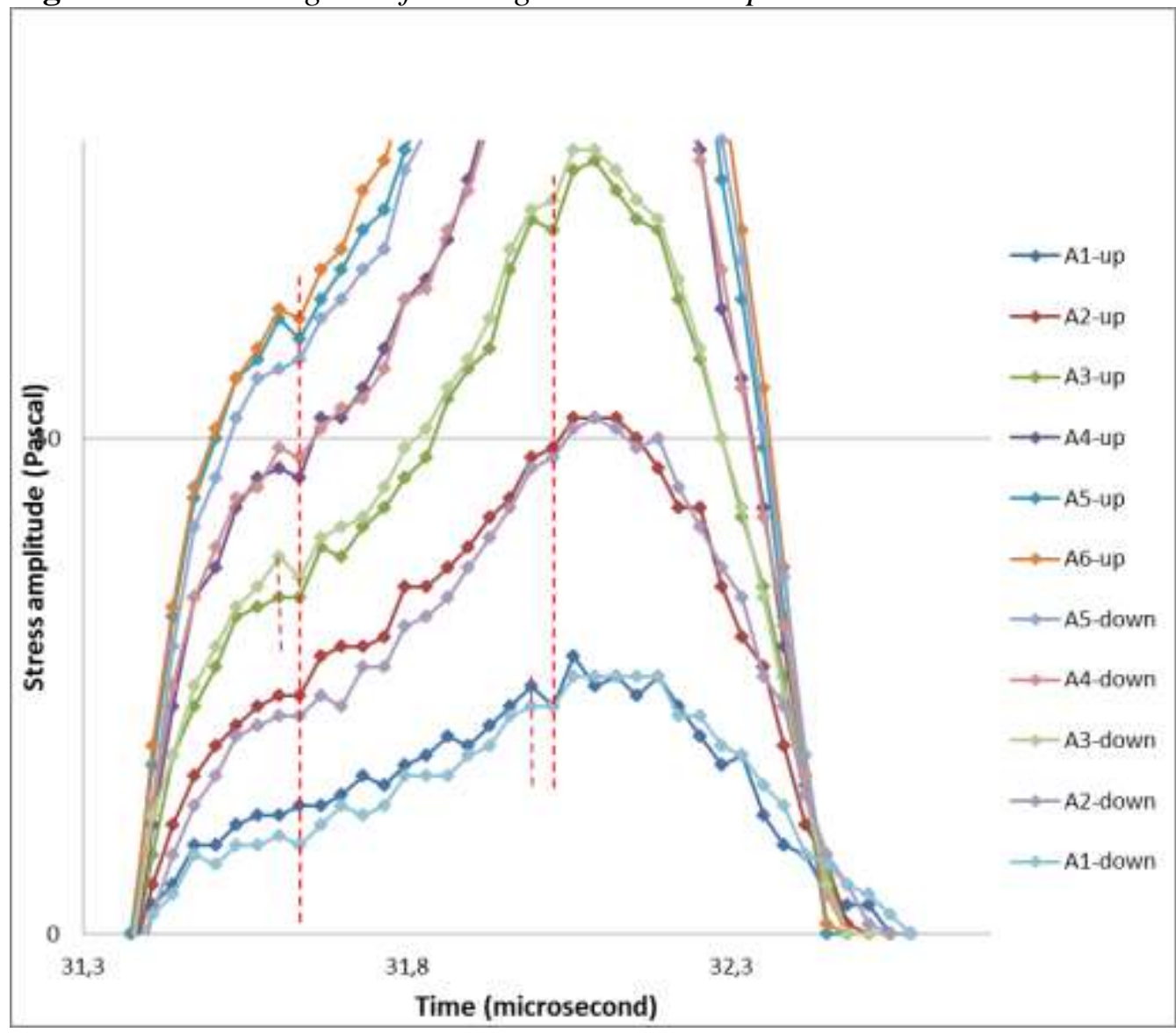

\section{Discussion}

In the submitted dynamic experiments the following step in the microplasticity process comprehension in a geologic medium has been made. Phenomenon mechanism is not yet completely been understood. The unusual deformation process detected in rocks in the small-strain range is of interest both in basic and applied sciences. The plasticity participation in the deformation process in rock was earlier been considered only for the regime of the large and moderate deformations.

It is necessary to mention that microplasticity in metals, alloys, and other solid states is well known (Nishino and Asano, 1996; Golovin et al., 2004). In crystalline materials plasticity is explained by the motion of dislocations in response to the applied stress. This implies that plasticity at the micro/nano scale has a discrete nature both in space and time. Modern data show that the stress-strain curve is formed by small jumps and plateaus that interrupt the "normal" stress-strain course. This phenomenon resembles the intermittent avalanches in the material (Zhou et al., 2010). At that, absolute strain values are within nanometers. Modern theories give a homogenized description of dislocation motion in the micro-scale (Berdichevsky, 2005; Kim et al., 2006). 
It is important to note that acoustic nonlinearity can be caused by a number of different sources.

There are theoretically well-founded data received with the help of the molecular dynamics method and the use of the micro structural model for a study of the micro-plastic regime of a stress-strain curve that explains the strain burst and avalanche phenomenon (Derlet and Maaf, 2013). The parameters of this model, along with the mobile dislocation density, admit a diversity of micro-plastic behavior involving intermittent plasticity in the avalanche-like process. Stress plateaus indicating strain burst activity is clearly visible on the stress-strain curves. Data obtained in our work are closely related to the results obtained for other solids but these have own distinctive manifestations. On Figures 1, 2, 3 presented in our paper, we distinctly see the intermittent inelasticity manifestations in the form of the stress plateau and the stress drops that predicted by theoretical works (Derlet and Maaf, 2013). Besides in this work, the interchangeability effect of discrete-inelastic manifestations is also described. The microhysteresis on a separate part of the $\sigma(\mathrm{t})$ curve (see Figure 2 ) with the participation of a viscoelastic and microplastic process is not yet fully understood. There is an assumption that the stress pulse during its propagation passes through some parts in the rock, which have sharply differing geomechanical properties. This anomalous microvolume of the rock has a unequal modulus of elasticity for an upward and downward stress path, that determines the mismatch of these courses and the emergence of a hysteresis loop. This result is representative, at least, there is a quasi-microplasticity, and it is confirmed by previous studies (Mashinskii, 2014). This work also shows the effect of quantization time on signal recording. The increase in the quantization time can lead to some loss of information, but does not exclude the very existence of discrete inelasticity.

In Earth science, this knowledge is almost absent. Heretofore the direct proofs of rock microplasticity have been gained only for quasi-static deformation on the samples of rocks (Mashinsky, 1994), therefore any dynamic study especially in natural conditions is the considerable advancement. It is ascertained that microplasticity manifestation character and degree of pulse transformation depend on the amplitude level, i.e. the stress intensity. Could also say, there is the effect of the stress rate. The plateaus on the seismic/acoustical trace mean the short-duration interruption in the stress course (Sapozhnikov et al., 1996; Mashinskii, 2012; 2016).

The possible reason of the amplitude effect is the various sources of multilevel microplasticity that begin to act when the stress amplitude increases. With increasing amplitude, the contribution change of elastic and microplastic elements occurs. If microplasticity dominates, the wave front becomes more flat and on the contrary when the elasticity dominates, the wave front becomes steeper. The microplasticity contribution variation with change of the stress amplitude was established in the work (Mashinskii, 2005b). Total strain consists of three main components: $\varepsilon_{i}=\varepsilon_{i-e}+\varepsilon_{v-e}+\varepsilon_{\mu}$, where the component $\varepsilon_{i-e}$ represents an ideal elastic strain, the $\varepsilon_{\mathrm{v}-\mathrm{e}}$ is viscoelastic strain and the $\varepsilon_{\mu}$ is microplastic strain that is the amplitude-dependent deformation. 
There is reason to believe that character and intensity of microplasticity manifestation depend on rate of deformation (or rate of loading/unloading). One example of this effect can be seen in Figure 3 where the pulses tops are shown for the amplitudes $A_{1}$ and $A_{2}$ in loams. In the region of the pulse maximum, it is immediately obvious that the rate of stress decreases, and the number of plateaus and their length increase. Perhaps this is due to the fact that more high stress rate does not allow bring into action some microplasticity sources whereas at low rate of stress these sources easily action. Microplasticity sources (for example, movable dislocations or others) can have the multilevel thresholds for inclusion of microplastic flow.

In physical literature, there are numerous theoretical and experimental data relating to our theme. In some solid materials, the deformation stops caused by microplasticity are known (Smirnov et al., 2002). The yielding plateau of the stress-strain curve is detected in diverse solid states. This is "pop-in" and "popout effects" that act under loading and unloading, accordingly (Bradby and Williams, 2004). Plateau means the continuity interruption (discontinuity, burst) in the stress course. Such transition from an elastic to elastic-plastic behavior occurs repeatedly. Defects have a strong influence on the yielding behavior. These effects have the threshold nature. Pop-in effects were detected even in dislocation-free crystals (Lorenz et al., 2003). Such nonstandard inelastic effects are detected also in minerals (silica stishovite, silicon, sapphire, diorite, graphite, mica) in the range of deformations about $10^{-4}-10^{-3}$ (Sheng-Nian Luo et al., 2007). It is significant that the microplastic inelasticity in loams and siltstone can be activated in the small-strains range $\left(\sim 10^{-8}-10^{-5}\right)$. Our range of microplastic deformation is included in the range of deformations studied in solid state physics, micro/nano mechanics and other disciplines.

Theoretical study of the microplasticity effect in rocks was undertaken in the some works (Yarushina and Podladchikov, 2008; 2010; 2015). The research is conducted that shows It is possible also the combination of several known mechanisms, for instance, as the hybrid mechanism (Mashinskii, 2006).

\section{Conclusions}

Comparison of field and laboratory data shows that despite the difference in measurement conditions, the qualitative characteristics of microplasticity in both cases have a similar character. Complications of the pulse form due to microplasticity (as in other cases) are presented in the form of the stress plateau and the stress drop. This result obtained with the help of an alternative method confirms the reliability of the data.

The detection of the nonstandard inelastic property in rocks gives the newest contribution to the understanding of the deformation process in the Earth and in particular the wave propagation dynamics. Well-known wave attenuation mechanism can be improved by inclusion in the rheological model of the new element connected with microplastic inelasticity. Established earlier fact of the amplitude dependence of seismic parameters is at least bound with microplasticity. 
Short-term interruption of elastic deformation caused by dynamic force testifies about microplasticity participation in the deformation process as additional mechanism. Such process can occur also in geodynamics, seismology etc. Presence of threshold effect due to microplasticity can be important in numerous areas of the science and engineering.

Microplasticity mechanisms, its manifestations in geological materials are not yet completely understood. This mechanism can be like as in solids (dislocations motion, other known mechanisms) or to be totally other mechanism. Any microstructural defects (inhomogeneities, germinal cracks, gaseous-liquid phase in pores of quartz crystal) create favorable conditions for the manifestation of intermittent inelasticity, including microplasticity. The question of microplasticity mechanism requires a further clarification. The new type of rock's inelasticity affects the little-known inelastic processes of the smallamplitude wave propagation in natural materials. Knowledge of inelastic processes is useful not only for the development of the theory, but also for the creation on this basis of new search attributes that enhance the effectiveness of seismic and acoustic methods.

\section{Acknowledgments}

The author thanks Dr. G.V. Egorov for the help in experiments and discussion of this work.

\section{References}

Adushkin V. V., Kostuchenko V. N., Kocheryan G. G., Pavlov D. V., 1999. Nonlinear deformation of rock masses on transformation of low-amplitude seismic waves. Doklady Earth Sciences, Vol. 368, N 1, pp. 958-961.

Berdichevsky Victor L., 2005, Homogenization in micro-plasticity. Journal of the Mechanics and Physics of Solids, 53, p. 2457-2469.

Braccini, S. et al., 2000. The maraging-steel blades of the Virgo super attenuator. Meas. Sci. Technol., 11, 467-476.

Bradby J. E. and Williams J. S., 2004, Pop-in events induced by spherical indentaton ib compound semiconductors. J. Mater. Res., 19, No 1, 380-386.

Derlet P. M., Maaf R., 2013, Micro-plasticity and intermittent dislocation activity in a simplied micro structural model, arXiv:1205.1486v2 [cond- mat.mtrl-sci] 8 Feb 2013, p. 1-33.

Golovin, I. S., Sinning, H. -R., Goken, J., Riehemann, W., 2004. Fatigue-related damping in some cellular metallic materials. Materials Science and Engineering, A 370, 537-541.

Guyer, R. A., McCall, K. R. and Boitnott, G. N., 1995. Hysteresis, Discrete Memory and Nonlinear Wave Propagation in Rock: a New Paradigm. Phys. Rev. Lett., Vol. 74: 3491-3494.

Guyer, R. A., and P. A. Johnson, Nonlinear mesoscopic elasticity: Evidence for a new class of materials, Physics Today, 52, 4, 30-36, 1999. 
Johnston, D. H., Toksoz, M. N., 1980. Thermal cracking and amplitude dependent attenuation. Journal of Geophysical Research, 85, 937-942.

Kim J. -Y., Qu, J., Jacobs L. J., Littles J. W., and M. F. Savage, 2006, Acoustic Nonlinearity Parameter Due to Microplasticity. Journal of Nondestructive Evaluation, Vol. 25, No. 1, p. 29-37.

Lorenz D., Zeckzer A., Hilpert U., and P. Grau, 2003, Pop-in effect as homogenous nucleation of dislocationsduring nanoidentation. Physical Review, B 67, 172101.

Mashinskii E. I. and D'yakov G. N., 1999. Amplitude-dependent attenuation of pulse signals in rocks. Izvestiya Phys. Solid Earth, 35, 937-940.

Mashinskii, E., Koksharov, V. Z., Nefedkin, Yu. A., 1999. Amplitude-dependent effects in the range of small seismic strains. Russian Geology and Geophysics, 40, 611-618.

Mashinskii, E. I., 2004. The variants of the strain-amplitude dependence of elastic wave velocities in the rocks under pressure. Journal of Geophysics and Engineering 1, 295-306.

Mashinskii, E. I., 2005a. Experimental study of the amplitude effect on wave velocity and attenuation in consolidated rocks under confining pressure. Journal of Geophysics and Engineering 2, 199-212.

Mashinskii, E. I., 2005b. Non-linear stress-strain relation in sedimentary rocks and its effect on seismic wave velocity. Geophysica (Finland), 41 (1, 2), 3-17.

Mashinskii, E. I., 2006. Nonlinear amplitude-frequency characteristics of attenuation in rock under pressure. Journal of Geophysics and Engineering, 3, 291-306.

Mashinskii, E. I., 2007a. Effect of Strain Amplitude on the Relaxation spectra of attenuation in the dry and saturated sandstone under pressure. Journal of Geophysics and Engineering, 4, 194-203.

Mashinskii, E. I., 2007b. Amplitude-dependent effects of longitudinal seismic wave propagation in the interhole space. Izvestya, Physics of the Solid Earth, 43 (8), 683-690.

Mashinskii, E. I., 2008. Amplitude-frequency dependencies of Wave Attenuation in Single-Crystal Quartz: Experimental Study. Journal of Geophysical Research, 113, B11304.

Mashinskii, E. I., 2010. Seismo-microplasticity phenomenon in the rocks. Natural Science, $2(3), 155-159$.

Mashinskii, E. I., 2012. Microplasticity effect in low-velocity zone induced by seismic wave. Journal of Applied Geophysics, 2012, 83, p. 90-95.

Mashinskii, E. I., 2014. Elastic-microplastic nature of wave propagation in the weakly consolidated rock. Journal of Applied Geophysics, 2014, 101, p. 11-19.

Mashinskii, E. I., 2016. Dynamic microplasticity manifestation in consolidated sandstone in the acoustical frequency range. Geophysical Prospecting. Article first published online : 6 MAR 2016, DOI: 10.1111/1365-2478.12368.

Mashinsky, E. I., 1994. Quasi-micro-plasticity processes and nonlinear seismicity. Physics of the Solid Earth, 30, 97-102.

Mavko, G. M., 1979. Friction Attenuation: An Inherent Amplitude Dependence. Journal of Geophysical Research 84 (9), 4769-4775.

McCall, K. R. and Guyer, R. A., 1994. Equation of State and Wave Propagation in Hysteretic Nonlinear Elastic Materials. J. Geophys. Res., Vol. 99, No B12: 2388723897.

Nishino, Y., Asano, S., 1996. Amplitude-dependent internal friction and microplasticity in thin-film materials. Journal de Physique IV, 6, 783-786.

Ostrovsky, L. A., Johnson, P. A., 2001. Dynamic nonlinear elasticity in geomaterials. La Rivista del Nuovo Cimento 24, 4, 7. 
Sapozhnikov, K. V., Vetrov, V. V., Pulnev, S. A., Kustov, S. B., 1996. Acoustopseudoelastic effect and internal friction during stress-induced martensitic transformations in Cu-Al-Ni single crystals. Scripta Materialia., 34 (10), 1543-1548.

Sheng-Nian Luo, J. G. Swadener, Chi Ma, Oliver Tschauner, 2007. Examining crystallographic orientation dependence of hardness of silica stishovite. Physica, B 390, 138-142.

Smirnov, B. I., Shpeizman, V. V., Peschanskaya, N. N., Nikolaev, R. K., 2002. Effect of magnetic field on microplastic strain rate for $\mathrm{C}_{60}$ single crystals. Physics of the Solid State, 44 (10), 2009-2012.

Tutuncu, A. N., Podio, A. L., Sharma, M. M., 1994. An experimental investigation of factors influencing compressional- and shear-wave velocities and attenuations in tight gas sandstones. Geophysics, 59 (1), 77-86.

Winkler, K. W., Nur, A., Gladwin, M., 1979. Friction and seismic attenuation in rock. Nature 274, 528-531.

Xu, H., Day, S.M., Minster, J. -B. H., 1998. Model for Nonlinear Wave Propagation Derived from Rock Hysteresis. Measurements Journal of Geophysical Research, 103, (B 12), 29,915-29,929.

Yarushina, V. M., Podladchikov, Y. Y., 2008. Micriscale yielding as mechanism for low-frequency imtrinsic seismic wave attenuation. $70^{\text {th }}$ EAGE Conference \& Exhibition - Rome, Italy, 9-12 June 2008.

Yarushina, V. M., Podladchikov, Y. Y., 2010, Plastic yielding as a frequency and amplitude independent mechanism of seismic wave attenuation. Geophysics, 75, 3, 51-63.

Yarushina, V. M., and Y. Y. Podladchikov, 2015, (De)compaction of porous viscoelastoplastic media: Model formulation, J. Geophys. Res. Solid Earth, 120, doi:10.1002/2014JB011258.

Zaitsev, V. Yu., Nazarov, V. E., Talanov, V. I., 1999. Experimental Study of the selfaction of seismoacoustic waves. Acoustic Physics, 45 (6), 720-726.

Zhou Caizhi, Biner S. Bulent, LeSar Richard, 2010, Discrete dislocation dynamics simulations of plasticity at small scales. Acta Materialia, 58, p. 1565-1577. 